\title{
Uso de modelos tridimensionais no ensino da Geografia Física e Paleontologia
}

\section{The use of three-dimensional models for teaching Physical Geography and Palaeontology}

\section{El uso de modelos tridimensionales para la enseñanza de Geografía Física y Paleontología.}

\begin{abstract}
Luiz Eduardo Panisset Travassos- luizepanisset@gmail.com Doutor em Carstologia pela Universidade de Nova Gorica (Eslovênia). Professor permanente do PPGeo da PUC Minas e professor colaborador do PPGeo da UFMG Avenida Dom José Gaspar 500, Coração Eucarístico, Belo Horizonte, Gerais. CEP: 30535901 Orcid: https://orcid.org/o0o0-0001-6264-2429
\end{abstract} Lucas Diniz de Arêda- lucasareda@gmail.com
Bolsista CAPES. Doutorando em Geografia pelo Programa de Pós-Graduação em
Geografia da PUC Minas
Avenida Dom José Gaspar 500, Coração Eucarístico, Belo Horizonte,
Minas Gerais. CEP: 30535901
Orcid : $\underline{\text { https://orcid.org/oooo-0003-1518-8168 }}$

Pedro Oliveira Paulo-pedro.paulo@ueg.br Biólogo, Doutor em Geologia Regional/Geociências e Meio Ambiente pela Universidade Estadual Paulista 'Júlio de Mesquita Filho', Campus de Rio Claro/SP e Docente de Geologia e Paleontologia da Universidade Estadual de Goiás, Câmpus Central - Sede. Br 153, No 3105, Caixa Postal 459, Anápolis, Goiás CEP 75132-400 Orcid : https://orcid.org/oooo-0002-2972-8455

\section{Resumo}

O presente trabalho tem como objetivo principal demonstrar a aplicabilidade da impressão $3 \mathrm{D}$ de modelos digitais do terreno e paleontológicos para serem utilizados na Educação Básica e Superior, em especial, nas disciplinas de Geografia Física, Geologia e Paleontologia. Os procedimentos metodológicos incluíram revisão de literatura, seleção das feições e materiais fósseis ou reconstruções paleontológicas a serem impressas e escolha de software para geração dos modelos digitais. Os resultados apontaram que a impressão em 3D demonstrou ser uma maneira rápida, econômica e eficaz de construir modelos digitais do terreno e modelos fósseis ou reconstruções de formas de vida do passado para serem utilizados no ensino de disciplinas das Ciências da Terra. Além disso, percebe-se que o uso dessa tecnologia pode dar mais materialidade a alguns conceitos dentro dos estudos cartográficos, geológicos,
\end{abstract}

geomorfológicos e paleontológico, propiciando, dessa forma, uma melhor compreensão de representação das características da natureza transformando o que é abstrato em algo mais concreto e de fácil acesso.

Palavras-chave: Impressão 3D; Modelos Digitais do Terreno; Recursos didáticos.

\begin{abstract}
The work's main objective is to demonstrate the applicability of $3 \mathrm{D}$ printing of digital terrain and paleontological models in Basic and Higher Education, especially in Physical Geography, Geology and Paleontology. The methodological procedures included a literature review, selection of features and fossil materials or paleontological reconstructions to be printed and choice of software for the generation of digital models. The results indicated that $3 \mathrm{D}$ printing was a
\end{abstract}


fast, economical and effective way to build digital models of the terrain and fossil models or reconstructions of past life forms, which can be used in Earth Science disciplines. In addition, it points out that this technology can provide a handleable resource within cartographic, geological, geomorphological and paleontological studies, thus providing a better understanding of the representation of nature's features, converting abstract information into concrete and easily accessible object.

Keywords: 3D printing; Digital Terrain Models; Didactic resources.

\section{Resumen}

El presente trabajo tiene como principal objetivo demostrar la aplicabilidad de la impresión 3D de terrenos digitales y modelos paleontológicos para ser utilizados en la Educación Básica y Superior, especialmente en las disciplinas de Geografía Física, Geología y Paleontología. Los procedimientos metodológicos incluyeron revisión de la literatura, selección de características y materiales fósiles o reconstrucciones paleontológicas a imprimir y elección de software para la generación de modelos digitales. Los resultados indicaron que la impresión $3 \mathrm{D}$ era una forma rápida, económica y eficaz de construir modelos digitales del terreno y modelos fósiles o reconstrucciones de formas de vida pasadas, que se pueden utilizar en las disciplinas de las Ciencias de la Tierra. Además, señala que esta tecnología puede proporcionar un recurso manejable dentro de los estudios cartográficos, geológicos, geomorfológicos y paleontológicos, proporcionando así una mejor comprensión de la representación de los rasgos de la naturaleza, convirtiendo una información abstracta en un objeto concreto y de fácil acceso.

Palabras clave: impresión 3D; Modelos digitales de terreno; Recursos didácticos. 


\section{Introdução}

Desde pelo menos a década de 8o, as tecnologias de impressão em três dimensões (3D) foram patenteadas e estão comercialmente disponíveis (HULL, 1986) no mercado. Apresentam, portanto, grande potencial tanto para desenvolver campos da ciência e tecnologia, quando os processos de ensinoaprendizagem na Educação Básica e Superior. Schelly et al. (2015) afirmam que as tecnologias de fontes abertas (open-source OS) são, atualmente, importantes ferramentas educacionais, pois seus custos reduzidos encaixam-se perfeitamente no cenário educacional de muitos países em especial, o Brasil.

Encontra apoio para essa afirmação o trabalho de Santana et al. (2016), que destacam a democratização dos processos de Manufatura Aditiva (Additive Manufacturing - AM) por extrusão, motivada pelo desenvolvimento das impressoras $3 \mathrm{D}$ de código aberto, que permitiu o uso dessas tecnologias também em ambientes domésticos, garantindo maior flexibilidade e possibilidades de adaptações. Além disso, Berman (2012) e Attaran (2017) afirmam que as impressões em três dimensões (3D) possibilitam customizações tanto de larga quanto de pequena escala a custos relativamente baixos, especialmente em países em desenvolvimento.

Em relação aos materiais didáticos, Gomes et al. (2005) afirmam que sua produção deveria fazer parte da formação dos professores de Geografia, Geologia, Biologia e, consequentemente, Paleontologia. Todavia, por diversos motivos (e.g. tempo, disponibilidade de recursos, laboratório apropriado etc.), isso não ocorre. Frequentemente o que existe é apenas a informação sobre as possibilidades e as facilidades do emprego dos materiais em sala de aula. Assim sendo, é importante a busca por instrumentos acessíveis e que facilitem o processo de ensinoaprendizagem, sejam por sequências didáticas ou por metodologias que abordem o Ensino por Investigação (SASSERON, 2018).

Terribili (2008) buscou verificar a cognição e aprendizado de estudantes de forma comparativa por meio de um teste realizado em duas turmas de pósgraduação na Universidade Estadual Paulista. No teste, os alunos deveriam decorar trinta palavras escritas em uma folha e reproduzi-las posteriormente. As palavras faladas, exibidas em uma projeção e escritas foram $29 \%$ mais armazenadas que as palavras que apenas foram faladas, e 15\% mais armazenadas que as palavras reproduzidas e vistas, o que mostra que a integração do uso dos sentidos é benéfica. As informações fazem mais sentido para o sujeito se ele vivenciar situações multissensoriais o máximo possível.

Deste modo, acredita-se que existe possibilidade de maior aproveitamento cognitivo com o uso didático de modelos tridimensionais de feições, fenômenos geológicos, geomorfológicos e paleontológicos, tornando possível levar à sala de aula elementos da paisagem natural.

Para o ensino de geomorfologia, tradicionalmente se usam imagens de satélite e fotografias. É inegável, contudo, o impulso dado às áreas associadas àgeomorfologia pelo estudo das formas do terreno de maneira mais concreta. Surgem, portanto, os MDT, ou Modelos Digitais do Terreno. De acordo comFelgueiras (1999), tais modelos representam o comportamento de um fenômeno que ocorre em uma região da superfície terrestre, produzidos a partir de valores de altitude do nível do solo. Tornaram-se populares nas geociências, pois permitem a derivação de diversos produtos tais como mapas de declividade,

TRAVASSOS, Luiz Eduardo Panisset; ARÊDA, Lucas Diniz de; PAULO, Pedro Oliveira. Uso de modelos tridimensionais no ensino da Geografia Física e Paleontologia. Boletim Alfenense de Geografia. Alfenas. v.1, n.2, p 63-75, 2021. ISSN: 2764-1422. DOI: https://doi.org/10.29327/243949.1.2-6 
curvas de nível e visualização 3D, por exemplo (BURROUGH, 1986; WEIBEL; HELLER, 1991; MACEDO et al., 2003; GROHMANN et al., 2008).

Para Carvalho e Latrubesse (2004), o uso de um MDT favorece os estudos geológicos e geomorfológicos, pois, como um produto digital, apresenta informações x, y e z (longitude, latitude e altitude). Seu objetivo é permitir análises quantitativas e qualitativas do terreno com o uso de programas específicos. Com os MDTs é possível a elaboração de perfis topográficos após serem traçados transectos na imagem, fato que destaca aspectos morfológicos da região em estudo.

No âmbito dos estudos paleontológicos, usualmente utilizavam-se a construção de réplicas e modelos em gesso de secagem rápida, produzidos em moldes de silicone comum e, em casos específicos, a utilização de agentes químicos catalizáveis, específicos como resina de laminação e polietilenoglicol. Com o advento de parcerias com institutos médicos que utilizam equipamentos de alta resolução, como Tomógrafos Computadorizados Multi-Slice, tornou-se possível realizar uma ampla variedade de estudos de material fossilífero ainda envolto em matriz sedimentar (AZEVEDO et al., 1994; 2009; 2014). Esta técnica permitiu a visualização de importantes e raros fósseis até então inédita, pois auxiliou na elaboração de estratégias de extração e preparo dos fósseis sem que haja perdas substanciais (SCHILLING et al., 2013). E fato de conhecimento entre os paleontólogos, os diversos processos tafonômicos que removem informações durante os diversos processos envolvidos na preservação destes restos, com especial destaque, na coleta, extração e preparação das amostras (SIMÕ̃S et al., 2014), que irão compor os acervos e as coleções tanto científicas, quanto didáticas.

Com a aquisição de Tomógrafos mais modernos por Instituições de Ensino e Pesquisa, como o Museu de Zoologia da USP - MZUSP, tornou-se mais frequente a geração de imagens em multi-slice ou por meio de cortes sequenciais do exemplar, ainda na matriz sedimentar envolvente, que são digitalmente manipuladas e que permitem a perfeita reconstrução do material incluso e, eventualmente, de materiais já preparados através de scanner. Adicionalmente, com o barateamento e a diversificação de marcas e modelos das impressoras em $3 \mathrm{D}$, estas imagens antes em corte, podem então compor o material fóssil original. Dessa forma, a produção de réplicas ou prototipagem em $3 \mathrm{D}$ de alta fidelidade com o especimen original, permite aos pesquisadores obter amostras de exemplares raros, localidades distantes, de intervalos de tempo variáveis, bem como, realizarem permutas, desde que os Museus, disponibilizem os arquivos gerados por meio destas e de outras técnicas a partir de seus respectivos acervos (ANDERSENSANTOS et al., 20013). Estes exemplares servem como ferramentas na difusão, divulgação e popularização da Ciência junto aos discentes de diversos níveis e modalidades, estudos científicos e exposições junto público de cada região, auxiliando na construção da identidade e respeito ao Patrimônio Geológico e Paleontológico nacionais.

Dessa forma, o presente trabalho tem por objetivo principal demonstrar a aplicabilidade da impressão $3 \mathrm{D}$ de modelos digitais do terreno para serem utilizados na Educação Básica e Superior, em especial, nas disciplinas de Geografia Física como Geomorfologia, Geomorfologia Estrutural e Hidrologia, entre outras. Adicionalmente, visa a utilização de prototipagem de modelos 
paleontológicos nos diversos níveis de Ensino, auxiliando no processo de ensinoaprendizagem e na divulgação e popularização da Ciência.

\section{Procedimentos metodológicos}

No âmbito da geografia física, após revisão de literatura, os autores decidiram realizar um recorte espacial que evidenciasse o território nacional. Para tanto, optaram por selecionar as feições geomorfológicas representativas que estão reunidas no livro Geomorfologia do Brasil (MAIO, 1987), publicado pelo IBGE e disponível gratuitamente em formato digital na página do Instituto.

Uma vez decidida a base teórica, buscou-se suporte em Hasiuk et al. (2017), que analisaram um aplicativo on-line de fonte aberta (TouchTerrain touchterrain.geol.iastate.edu), desenvolvido para simplificar a produção de arquivos *.obj ou *.stl para a produção de modelos digitais de elevação que possam ser impressos em 3D. No próprio TouchTerrain é possível utilizar a plataforma do GoogleMaps e selecionar uma escala para padronização dos modelos. Para essa pesquisa convencionou-se a utilização de altitude de $2 \mathrm{Km}$, perfazendo uma área de cerca de $400 \mathrm{Km}^{2}$.

No contexto paleontológico, diferentemente da abordagem da Geografia Física, optou-se pela utilização de imagens previamente escaneadas de exemplares paleontológicos disponíveis gratuitamente em sites, páginas de Instituições de Ensino Superior, Instituições de Pesquisa e Museus. Após a escolha e seleção dos arquivos de imagens compatíveis com a impressão em 3D, realizou-se a triagem para a obtenção específica de arquivos no formato * .stl para a produção dos modelos de teste. Em sequência a esta fase, seguiu-se à etapa de prototipagem de peças paleontológicas, tanto de fósseis propriamente ditos, quanto de exemplares reconstruídos com base em material coletado, tanto de vertebrados quanto de invertebrados. Dentre os vertebrados, destacam-se os crânios de fósseis como o Deinonychosaurus antirhopus Ostrom (1969), Velociraptor mongoliensis Osborn (1924) e Baurusuchus pachecoi Price (1945); dentre os invertebrados, o Anomalocaris sp., todos em escalas acessíveis ao tamanho e modelo da impressora (GTMax Core A1V2).

Figura 1 - Fluxograma metodológico.

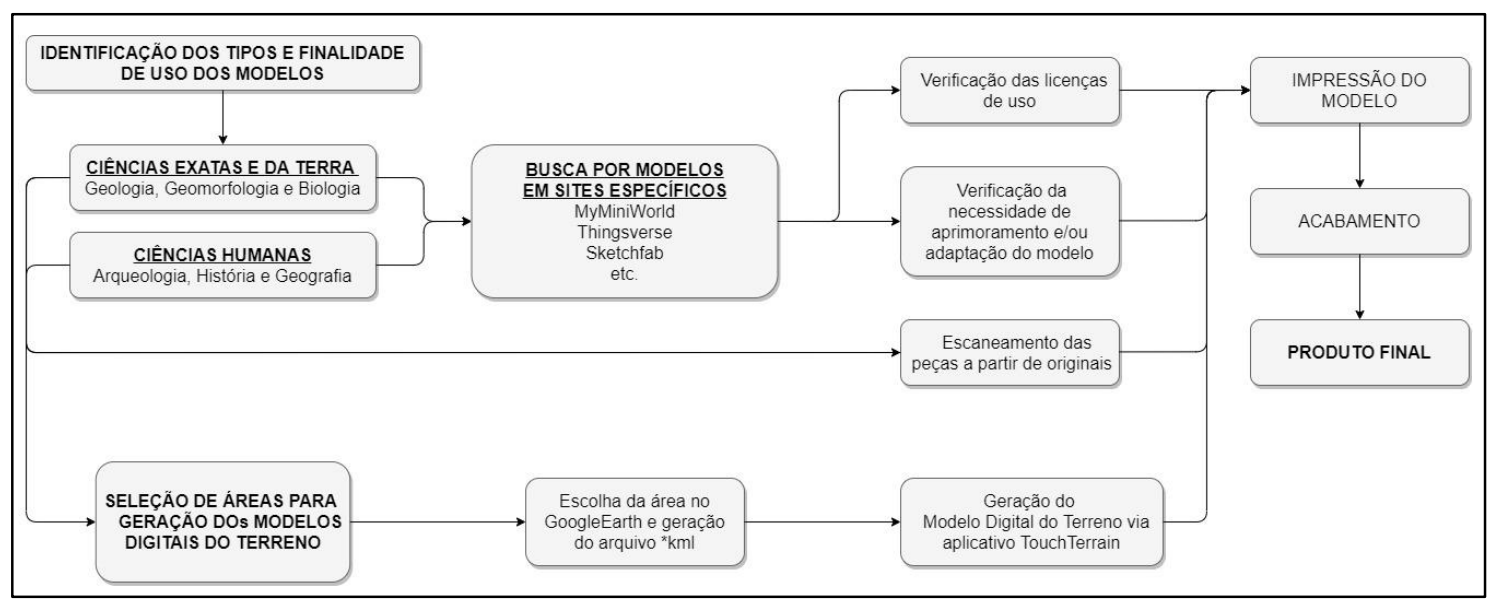

Fonte: Elaborado pelos autores 
Em relação ao software utilizado, optou-se por utilizar o Sliczr (RANELLUCCI, 2015), software de fonte aberta capaz de converter um modelo $3 \mathrm{D}$ para um arquivo possível de ser lido pela impressora, gerando os caminhos para o filamento e consequente conclusão do modelo impresso.

O material utilizado para confecção dos modelos foi o PLA (Poliácido Láctico), que, conforme Santana et al. (2016), é muito popular, principalmente entre entusiastas da impressão $3 \mathrm{D}$, uma vez que apresenta maior facilidade de impressão quando comparado a outros materiais disponíveis. Para este projeto, os parâmetros utilizados foram a temperatura do extrusor em $195^{\circ} \mathrm{C}$ e $80^{\circ} \mathrm{C}$ para a mesa que recebe o filamento derretido.

\section{Resultados e discussão}

Para esta proposta, seguindo a ordem dos capítulos do livro (MAIO, 1987), o primeiro modelo gerado foi o da vertente oriental da grande superfície em abóboda da Borborema, município de Alagoa Grande, Paraíba (Figura 2). De acordo com Maio (1987, p.14), as cristas constituem os núcleos mais elevados e resistentes do embasamento pré-cambriano formado, principalmente, por granitos, gnaisses e migmatitos, enquanto as áreas deprimidas são de micaxistos nos quais os rios se encaixam preferencialmente segundo os condicionantes estruturais e, secundariamente, orientados pelas heterogeneidades da litologia. Os abruptos relacionam-se a dois ciclos de erosão: 150 a 160 metros de altitude e 500 a 600 metros de altitude, encimados por superfícies de aplainamento.

Figura 2 - Vertente oriental da grande superfície em abóboda da Borborema, município de Alagoa Grande, Paraíba.

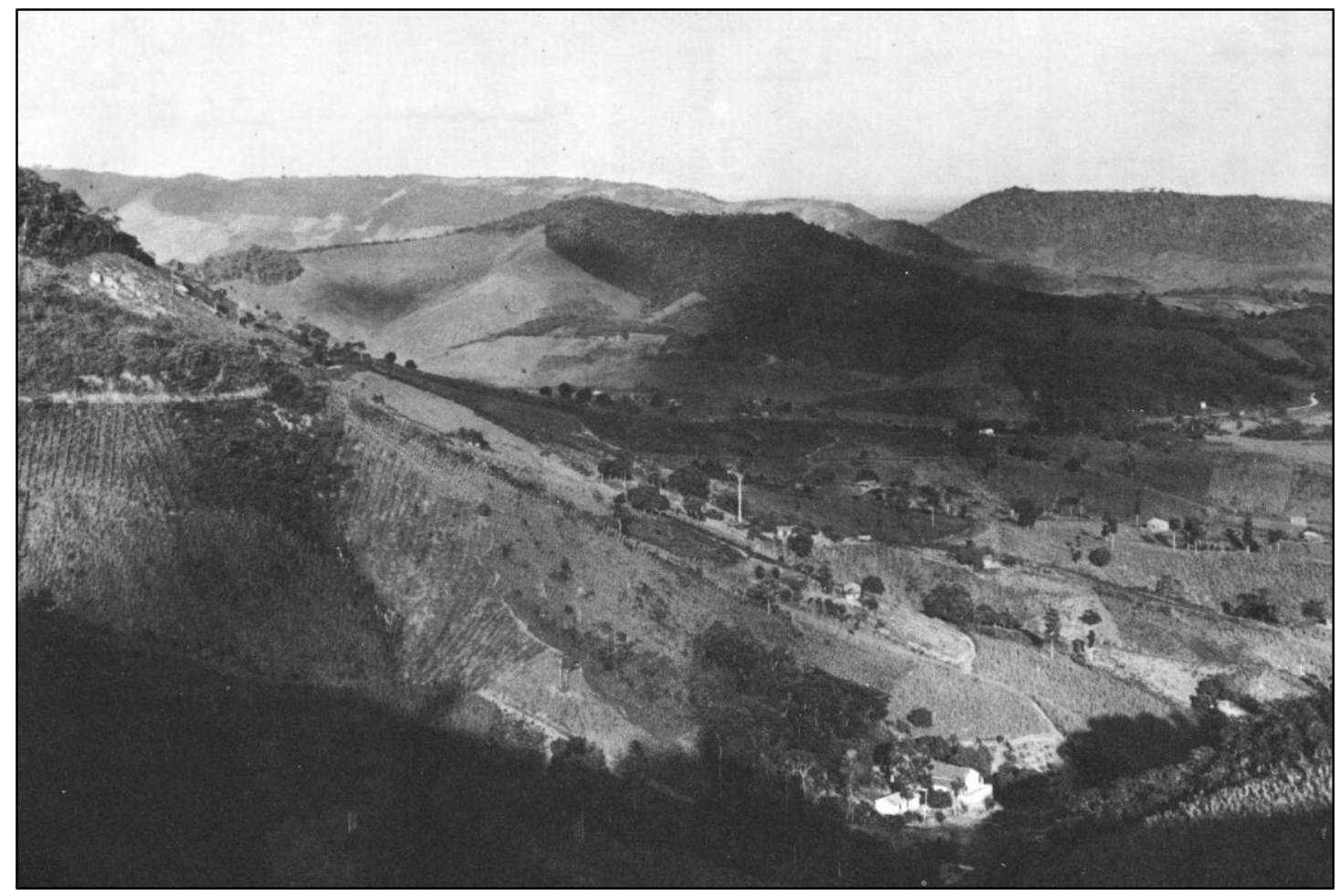

Fonte: MAIO, 1987, p.13 
A imagem fornecida pelo TouchTerrain apresenta resolução de 30 metros e pode ser identificada na figura 3. Verificadas as proporções entre as escalas horizontal e vertical e à luz das considerações feitas por Imhof (1982, p.207) sobre a adequação da representação aos preceitos cartográficos, decidiu-se por aplicar um exagero vertical de $150 \%$ (fator 1.5 no menu de opções do TouchTerrain) que foi padronizado para todos os demais modelos produzidos no âmbito deste projeto, independente da escala horizontal. Esse procedimento permitiu considerar com maior propriedade, em modelos de pequenas dimensões finais, a expressividade das diferenças altimétricas representadas em cada caso e, comparativamente, induz a uma apreciação dos diferentes produtos geomorfológicos a partir da ação de fatores endógenos e exógenos de formação e de evolução do relevo.

Após a geração do MDT em formato *.obj ou *.stl, trabalhou-se o arquivo no Slic3r, conforme figura 4. O modelo produzido (Figura 5) foi uma representação de $144 \mathrm{~cm}^{2}$ da área escolhida. Portanto, apresenta escala de 1:166.000.

Figura 3 - Imagem selecionada da área de $400 \mathrm{~km}^{2}$.

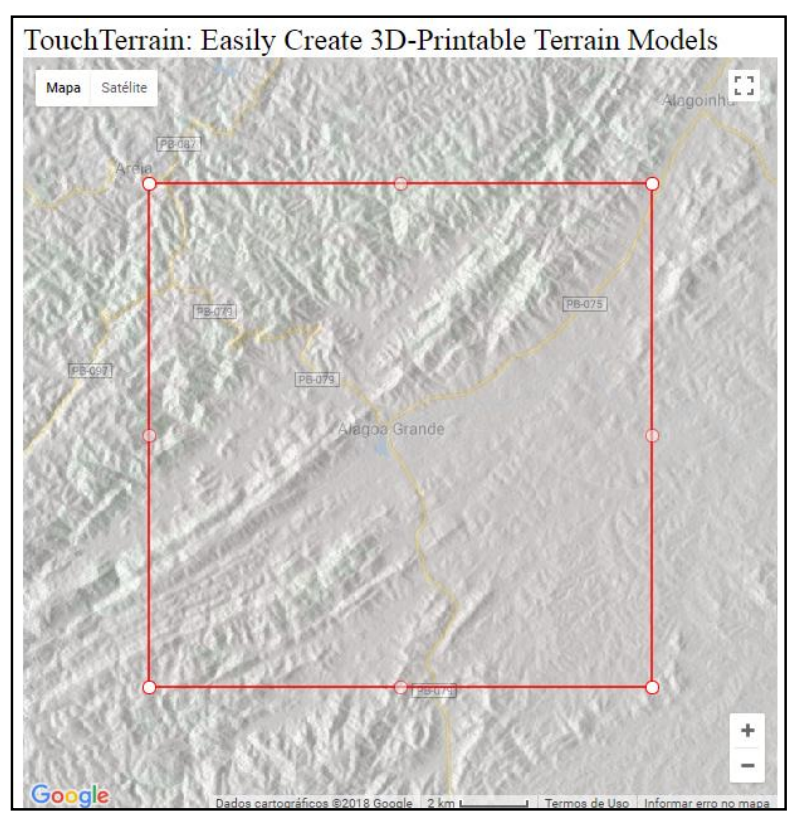

Fonte: TouchTerrain

Figura 4 - Modelo gerado pelo TouchTerrain, aberto no software Sliczr.

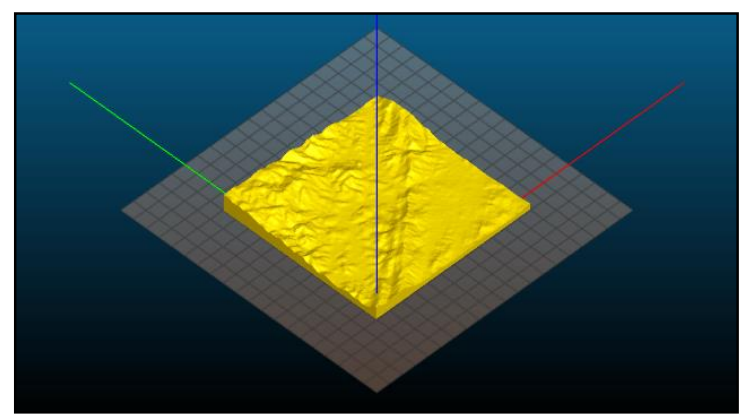

Fonte: Autores.

TRAVASSOS, Luiz Eduardo Panisset; ARÊDA, Lucas Diniz de; PAULO, Pedro Oliveira. Uso de modelos tridimensionais no ensino da Geografia Física e Paleontologia. Boletim Alfenense de Geografia. Alfenas. v.1, n.2, p. 63-75, 2021. ISSN: 2764-1422. DOI: https://doi.org/10.29327/243949.1.2-6 
Figura 5 - Aspectos do modelo digital do terreno. As fotos mostram o processo de impressão 3D em camadas e o preenchimento interno que confere resistência ao modelo, bem como o produto, sem pintura e pós-tratamento.

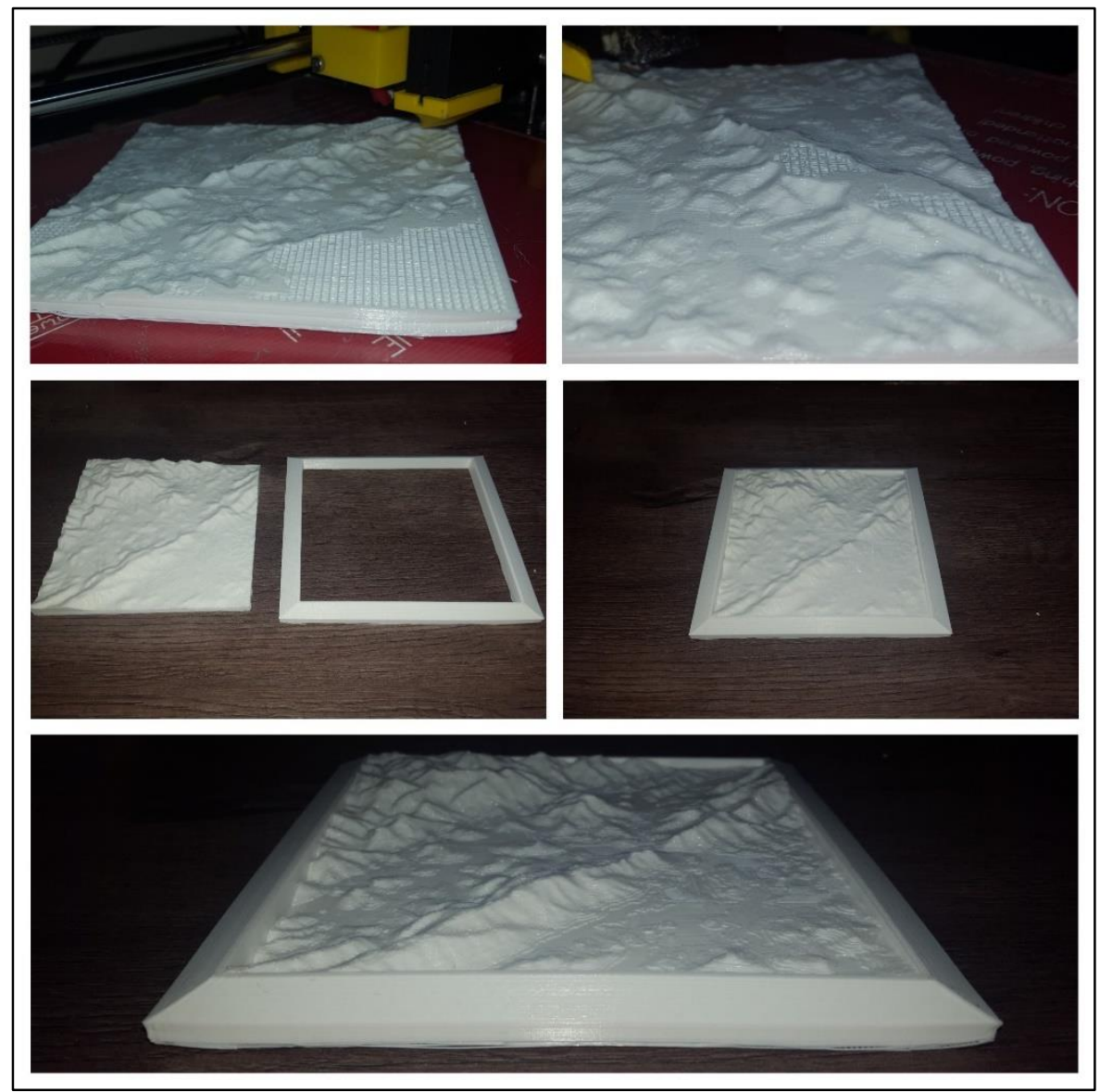

Fonte: Os Autores.

No âmbito dos modelos prototipados em Paleontologia, seguiram-se propostas similares às de Schilling et al. (2013) e Santos et al. (2003), no entanto, substituiu-se o uso do Tomógrafo Computadorizado para a geração de imagens, optando-se pelo uso de imagens disponíveis ou pela geração destas por meio de aparelho de scanner portátil. No processo de busca de imagens em formato * stl ou obj*, deu-se preferência àquelas de livre acesso ou disponibilizadas de modo gratuito por museus ou pesquisadores, sendo

Não houve a necessidade de tratamento prévio das imagens que comporiam os modelos, já que eram resultantes de acessos aos materiais originais feitos para fins de pesquisa e divulgação. Em se tratando de escalas, pelo fato de lidar com exemplares fósseis com grandes proporções anatômicas, optouse pelo cálculo das escalas que oferecessem o melhor custo-benefício, uma vez que, a prototipagem consome uma quantidade expressiva de energia elétrica. No caso de modelos prototipados de fósseis de tamanho pequeno a médio, optou-se pelas escalas de 1:1, como em Velociraptor mongoliensis (Figura 6a) e Deinoychus antirrhopus (Figura 6b2), bem como os modelos de exemplares de Anomalocaris sp. (Figura 6c), Hallucigenia sp. (Figura 6d), Pikaia gracilens (Figura 6e)e Compsognathus longipes (Figura 6f). 
Figura 6 - Modelos fósseis de tamanho pequeno a médio, com pintura e póstratamento. A) Velociraptor mongoliensis; B) Deinoychus antirrhopus. Na foto superior, o crânio original e na parte inferior, a réplica começando a ser pintada.

C) Processo de finalização do modelo representativo do Anomalocaris sp, com $70 \mathrm{~cm}$; D) Hallucigenia sp.; E) Modelo da espécie Pikaia gracilens; F) Modelo finalizado de um Compsognathus longipes.
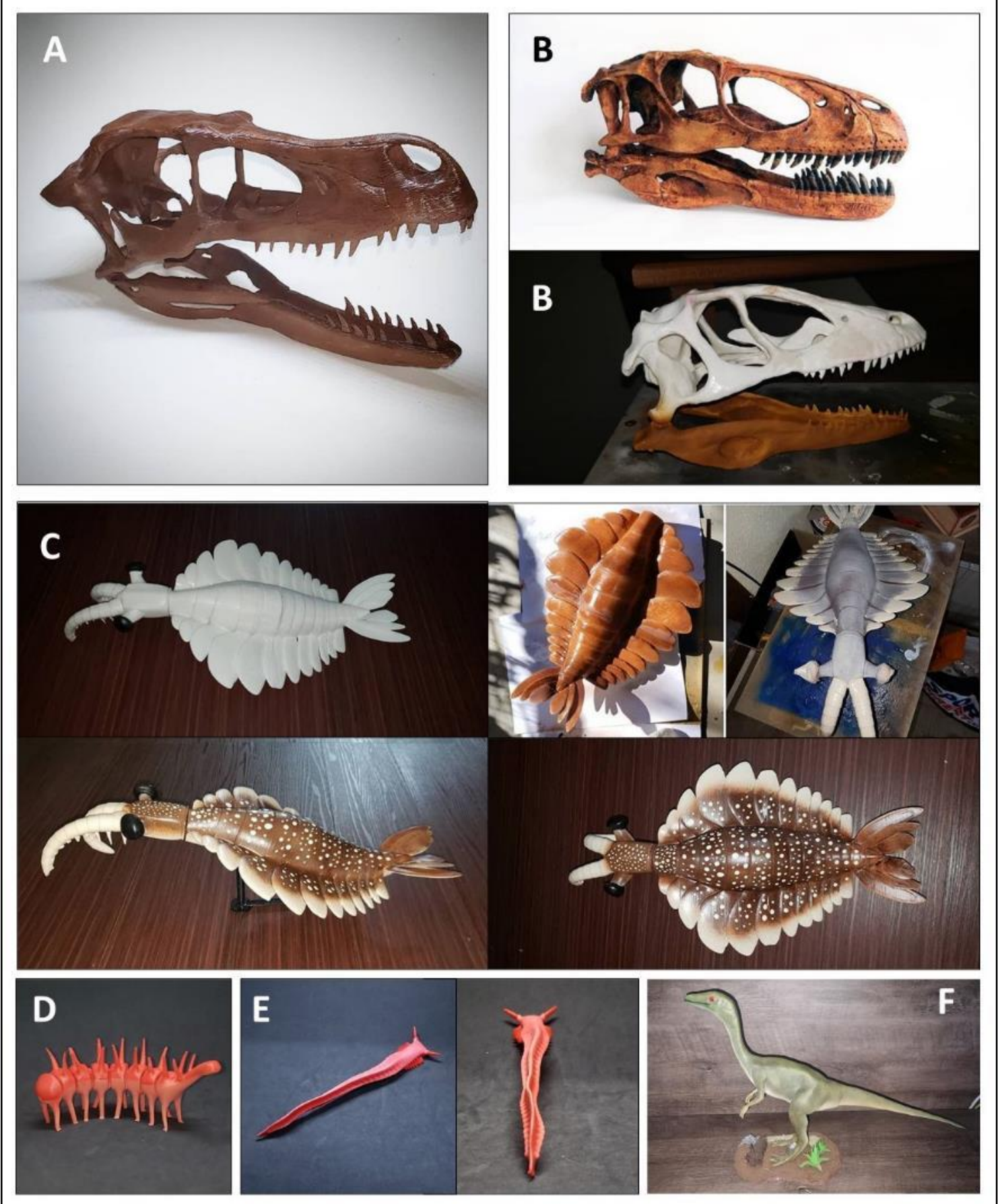

Fonte: Os Autores. 
Tanto os modelos de amostras fósseis produzidas, quanto os modelos em escala, representando os organismos em vida, necessitaram ser impressos em etapas, principalmente, devido às proporções da impressora e sem prejuízos para a qualidade dos produtos, que foram pintados com cores de análogos modernos.

Retornando para o ensino das Ciências da Terra, outros modelos populares são as Ilhas Vulcânicas do Havaí, esquemas para explicação das falhas, bem como esquema das camadas da Terra (Figura 7), pois, geralmente, são mencionado nos planos de ensino das disciplinas de geologia e geografia, por exemplo.

Figura 7 - Outros modelos que podem ser utilizados nas aulas de Geografia Física e Geologia. As fotos mostram, do topo para baixo, modelo que simula a formação de relevos estruturais (e.g. graben, horst e falhas), o interior da Terra e as Ilhas do Havaí. Todos como o produto, com pintura e pós-tratamento.

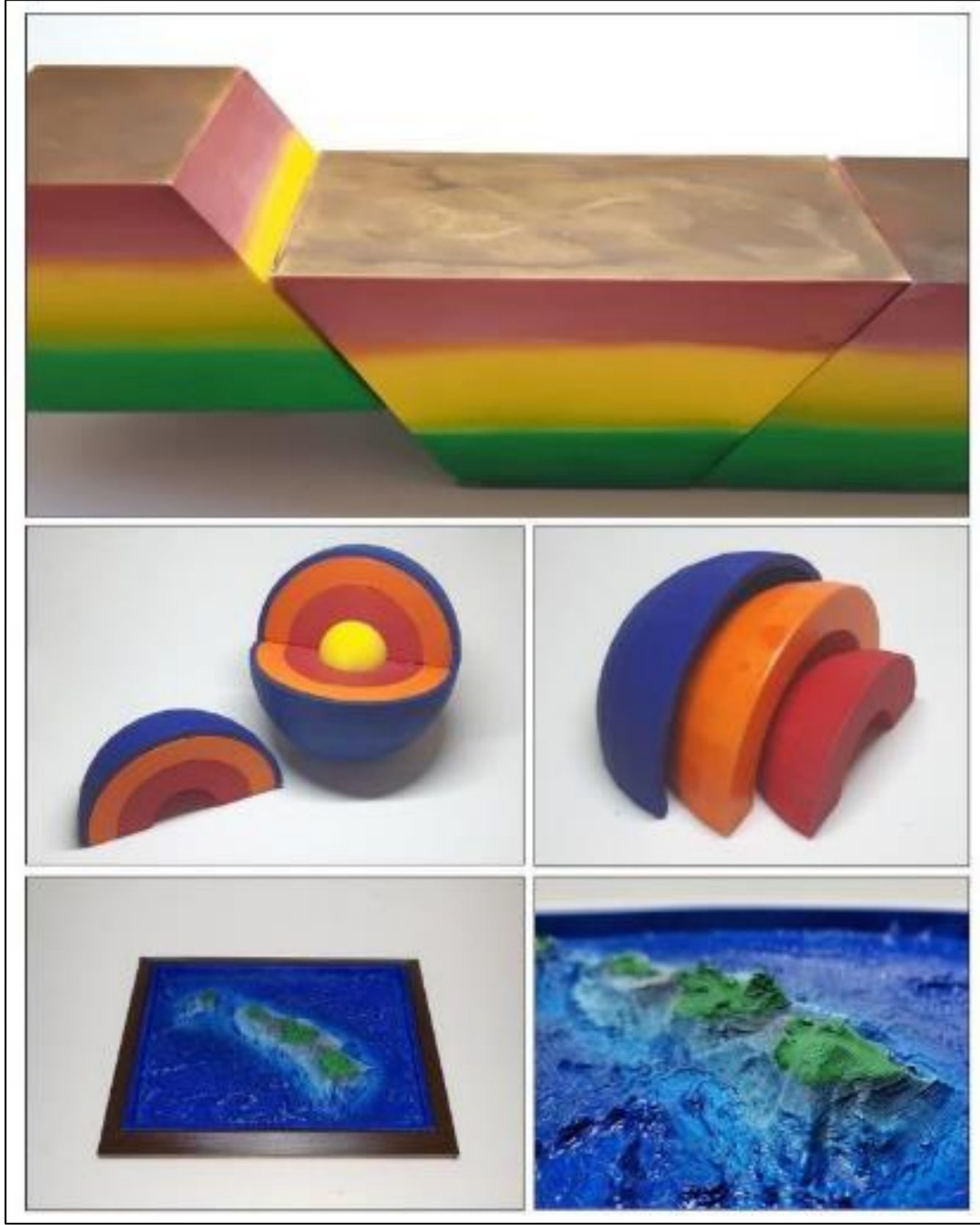

Fonte: Os Autores.

TRAVASSOS, Luiz Eduardo Panisset; ARÊDA, Lucas Diniz de; PAULO, Pedro Oliveira. Uso de modelos tridimensionais no ensino da Geografia Física e Paleontologia. Boletim Alfenense de Geografia. Alfenas. v.1, n.2, p 63-75, 2021. ISSN: 2764-1422. DOI: https://doi.org/10.29327/243949.1.2-6 


\section{Conclusão}

A impressão em 3D demonstrou ser uma maneira rápida, econômica e eficaz de construir modelos digitais do terreno e modelos paleontológicos para serem utilizados no ensino de disciplinas das Ciências da Terra. Observam-se mínimas imperfeições (facilmente corrigidas no pós-tratamento da peça) e pouco desperdício de material.

O uso dessa tecnologia pode dar mais materialidade a alguns conceitos dentro dos estudos cartográficos, geológicos, e geomorfológicos e paleontológicos, propiciando, dessa forma, uma melhor compreensão de representação das características da natureza, transformando o que é abstrato em algo mais concreto e passível de toque auxiliando, também, alunos com baixa visão.

A utilização da impressora 3D para construção de modelos abre caminho para a elaboração de exemplos geomorfológicos e paleontológicos que podem propiciar melhor entendimento das diferentes formas de relevo e dos variados tipos de organismos do passado geológico da Terra, não acessíveis diretamente. O contato e manuseio das mais variadas feições do relevo pode melhorar o entendimento do significado geomorfológico por parte do aluno. No âmbito paleontológico, permite aos alunos acessar amostras de organismos normalmente depositados em coleções distribuídas pelo mundo. Dessa forma, a prototipagem de materiais em geociências, torna um fator decisivo para $o$ aprendizado, já que pode reduzir ou eliminar dúvidas apresentadas pelos alunos.

Cabe destacar, ainda que este não seja o cerne deste trabalho, as possibilidades da materialização dos modelos criados, além de ampliar as possibilidades de ensino-aprendizagem, potencializam o alcance de um público que por vezes fica à margem de alguns processos de aprendizagem como aqueles que não conseguem enxergar ou apresentam alguma dificuldade. Os modelos impressos em 3D podem auxiliar na diminuição dos abismos existentes entre os alunos e os conceitos relacionados às questões espaciais no âmbito das Ciências da Terra, tão mais complexos quando não há compatibilização de meios pedagógicos para atingir esse igualmente importante grupo.

\section{Referências}

ANDERSEN, A. F.; CHAPMAN, R. E.; DICKMAN, J. Using prototyping technology in vertebrate palaeontology. Journal of Vertebrate Paleontology, v. 21, n. 3, 28A. 2001

ATTARAN, M. The rise of 3 -D printing: The advantages of additive manufacturing over traditional manufacturing. Business Horizons, v. 60, n. 5 , p. 677-688, 2017.

AZEVEDO, S. A. K.; SILVA, J. P.; SILVA, V. G.; FERIGOLO, J. O uso de tomografia computadorizada na Paleontologia de Vertebrados. Anais da Academia Brasileira de Ciências, v. 66, n. 2, p. 187-193, 1994. 
AZEVEDO, S. K; CARVALHO, L. B. O uso de tomografia computadorizada no estudo de vertebrados fósseis do Museu Nacional/UFRJ, p.1-32, In: WERNER Jr., H; LOPES, J. Tecnologias 3D: paleontologia, arqueologia e fetologia. Rio de Janeiro: Revinter, 2009. 190 pp.

AZEVEDO, S. A. K. ; CARVALHO, L. B.; SANTOS, D. D. Metodologias digitais aplicadas ao estudo de vertbrados. In: CARVALHO, I. S. Paleontologia: Conceitos e Métodos. Rio de Janeiro: Interciência, 2014. p. 445-450.

BERMAN, B. 3-D printing: The new industrial revolution. Business Horizons, v.55, n.2, p. 155-162, 2012.

BROUGH, P. A. Principle of geographical information systems for land resources assessment. Geocarto International, v.1, p. 54-54, 1986.

CARVALHO, T.M.; LATRUBESSE, E.M. Aplicacão de modelo digitais do terreno (MDT) em análises macrogeomorfológicas: o caso da bacia hidrográfica do Araguaia. Revista Brasileira de Geomorfologia, v.5, p.85-93, 2004.

FELGUEIRAS, C.A. Análises sobre modelos digitais de terreno em ambiente de sistemas de informações geográficas. São Paulo: DPI/INPE, 1999.

GOMES, P. C. C. Cenários para a Geografia: Sobre a espacialidade das imagens e suas significações. Espaço e Cultura: pluralidade temática. Rio de Janeiro: EDUERJ, p. 187-210, 2007.

GROHMANN, C. H.; RICCOMINI, C.; DOS SANTOS STEINER, S. Aplicações dos modelos de elevação SRTM em geomorfologia. Revista Geográfica Acadêmica, v.2, n.2, p. 73-83, 2008.

HARDING, C.; HASIUK, F. TouchTerrain: Easily Create 3D-Printable Terrain Models. Dept. of Geological and Atmospheric Sciences, Iowa State University. Disponível em: <http://touchterrain.geol.iastate.edu/>. Acesso em o5 mai. 2018.

HASIUK, F.J. et al. TouchTerrain: A simple web-tool for creating 3D-printable topographic models. Computers \& Geosciences, v. 109, p. 25-31, 2017.

HULL, C.W. Apparatus for production of three-dimensional objects by stereolithography. U.S. Patent n. 4,575,330, 11 Mar. 1986. Disponível em: $<$ https://patentimages.storage.googleapis.com/5c/ao/27/e49642dab9gcf6/US 4575330.pdf> Acesso em 22 mai.2018

IMHOF, E. Cartographic relief presentation. Berlin: Walter de Gruyter, 1982.

ISHUTOV, S. et al. Three-dimensional printing for geoscience: Fundamental research, education, and applications for the petroleum industry. AAPG Bulletin, v. 102, n. 1, p.1-26, 2018. 
KOSTAKIS, V.; NIAROS, V.; GIOTITSAS, C. Open source 3D printing as a means of learning: An educational experiment in two high schools in Greece. Telematics and informatics, v. 32, n. 1, p. 118-128, 2015.

MACEDO, M. de L. de A. et al. Geração de modelo digital de terreno a partir de mapas digitais $3 \mathrm{~d}$ : estudo de caso visando garantir o contexto geomorfológico com redução dos dados amostrais. Boletim de Ciências Geodésicas, v.7, n. 2, p.105-119, 2003.

MAIO, C.R. Geomorfologia do Brasil: fotos e comentários. 3.ed. Rio de Janeiro: IBGE, 1987. Disponível em: <https://biblioteca.ibge.gov.br/ visualizacao/livros/liv81454.pdf.> Acesso em: 15 fev.2018.

RANELLUCCI, A. Slic3r: G-code generator for 3D printers, 2015.

SANTANA, L. et al. Avaliação da composição química e das características térmicas de filamentos de PLA para impressoras $3 \mathrm{D}$ de código aberto. In: CONGRESSO NACIONAL DE ENGENHARIA MECÂNICA, 9, 2016. Anais... Fortaleza, 2016.

SASSERON, L. H.; CARVALHO, A.M.P. Alfabetização científica: uma revisão bibliográfica. Investigações em Ensino de Ciências, Porto Alegre, v. 16, n. 1, p. 59-77, 2018.

SCHELLY, C. et al. Open-source 3-D printing technologies for education: Bringing additive manufacturing to the classroom. Journal of Visual Languages \& Computing, v. 28, p. 226-237, 2015.

SCHILLING, R.; JASTRAM, B.; SCHWARZ-WINGS, D.; ISSEVER, A. Reviving the dinosaur: Virtual reconstruction and three-dimensional printing of a dinosaur vertebra. Radiology, v. 270, n. 3, p. 864-871, 2014.

SIMÕES, M. G.; RODRIGUES, S. CO.; BERTONI-MACHADO, C.; HOLZ, M. Tafonomia: Processos e Ambientes de Fossilização, In: CARVALHO, I. S. Paleontologia: Conceitos e Métodos. Rio de Janeiro: Interciência, 2014. pp. 19-52.

TERRIBILI, A. O uso dos sentidos na retenção da informação. In: SIMPEP, 15., 2008. Bauru. Anais... São Paulo, 2008

WEIBEL, R.; HELLER, M. Digital Terrain Modelling. In: MAGUIRE, D. J.; GOODCHILD, M. F.; RHIND, D. Geographical Information Systems. England: Longman, 1991. p. 269-294. 